\title{
Upregulation of TRIP13 promotes the malignant progression of lung cancer via the EMT pathway
}

\author{
RUJIAN LU ${ }^{1 *}$, QIAN ZHOU ${ }^{2 *}$, LINLING JU $^{3}$, LIN CHEN $^{3}$, \\ FENG WANG $^{4}$ and JIANGUO SHAO ${ }^{3}$ \\ ${ }^{1}$ Department of Cardiothoracic Surgery, Nantong Third People's Hospital, Nantong University; \\ ${ }^{2}$ Department of Internal Medicine, Medical School of Nantong University; \\ ${ }^{3}$ Nantong Institute of Liver Disease, Nantong Third People's Hospital, Nantong University; \\ ${ }^{4}$ Department of Laboratory Medicine, Affiliated Hospital of Nantong University, \\ Nantong, Jiangsu 226006, P.R. China
}

Received February 13, 2021; Accepted May 26, 2021

DOI: $10.3892 / o r .2021 .8123$

\begin{abstract}
Lung cancer is the most common malignant tumor type and it is associated with poor prognosis. The identification of potential biomarkers is of great significance for the early diagnosis and treatment of lung cancer. Non-small cell lung cancer (NSCLC) is the most common pathological type of lung cancer. The present study aimed to investigate the mechanism via which thyroid hormone receptor-interacting protein 13 (TRIP13) participates in the malignant progression of NSCLC. Immunohistochemistry, reverse transcription-quantitative PCR and western blotting were used to assess the expression level of TRIP13. According to The Cancer Genome Atlas database, TRIP13 was upregulated in NSCLC tissues compared with adjacent normal tissues. Moreover, TRIP13 knockdown increased apoptosis, induced cell cycle arrest in the $\mathrm{S}$ phase and inhibited the proliferation, invasion and migration of H1299 cells in vitro. Furthermore, TRIP13 upregulation was closely associated with tumor metastasis via epithelial-mesenchymal transformation. In conclusion, TRIP13 could promote the malignant progression of lung cancer, and TRIP13 may be a potential biomarker for the early diagnosis and treatment of NSCLC.
\end{abstract}

Correspondence to: Dr Jianguo Shao, Nantong Institute of Liver Disease, Nantong Third People's Hospital, Nantong University, 60 Middle Qingnian Road, Nantong, Jiangsu 226006, P.R. China E-mail: shaojianguo4144@163.com

*Contributed equally

Abbreviations: TRIP13, thyroid hormone receptor-interacting protein 13; NSCLC, non-small cell lung cancer; TCGA, The Cancer Genome Atlas; RT-qPCR, reverse transcription-quantitative PCR; CCK-8, Cell Counting Kit-8; IHC, immunohistochemistry; SAC, spindle assembly checkpoint; EMT, epithelial-mesenchymal transformation

Key words: TRIP13, lung cancer, proliferation, apoptosis, EMT

\section{Introduction}

At present, lung cancer has the highest mortality rate among all malignant tumors (1). According to statistics, as of 2018, lung cancer accounted for $11.6 \%$ of all cancer cases and cancer-related mortality (2). It is well known that smoking, air pollution and chronic lung disease are all common causes of lung cancer $(3,4)$. Although early screening for lung cancer is widespread, most patients are diagnosed at the terminal stage and have a poor prognosis. Despite current popular targeted therapy and immunotherapy, it remains difficult to achieve satisfactory results $(5,6)$. Determining the stage of lung cancer is required for treatment but it also important for evaluating prognosis. Based on the 8th edition of the American Joint Committee on Cancer, the size of the tumor, number of lymph nodes and sites of distant metastasis are the three major factors for determining cancer stage (7). Lung cancer can be divided into non-small cell lung cancer (NSCLC) and SCLC, then NSCLC can be further characterized into lung adenocarcinoma, lung squamous cell cancer and large cell cancer (8). Moreover, NSCLC is the most common pathological type. At present, providing a novel target for the molecular diagnosis and treatment of NSCLC has become a hotspot in basic research.

Thyroid hormone receptor-interacting protein 13 (TRIP13) is one of the most important members of the ATPase family associated with various cellular activities (AAA+) ATPase family, which is associated with cell cycle checkpoint and DNA repair $(9,10)$. TRIP13 participates in numerous cellular physiological processes, including chromosome checkpoints, DNA break repair and chromosome synapsis (11). The spindle assembly checkpoint (SAC), which is closely associated with chromosome repair, ensures that all chromosomes produce bipolar spindle structures before mitosis occurs $(12,13)$. The participation of TRIP13 affects the formation of SAC structure. For example, Tao et al (14) reported that overexpression of TRIP13 promoted multiple myeloma cell proliferation by invalidating checkpoints via the Akt pathway. TRIP13 is an oncogene considered to be involved in the progression of multiple tumor types, such 
as hepatocellular carcinoma $(15,16)$, colorectal cancer $(17)$, bladder cancer (18) and prostate cancer (19), and is associated with a poor prognosis. Zhang et al (20) revealed that TRIP13 may promote the malignant progression of NSCLC and may be a potential target. Furthermore, TRIP13 knockdown could arrest lung cancer cells in the $\mathrm{G}_{2} / \mathrm{M}$ phase, and it could regulate the expression levels of genes associated with cell cycle checkpoints. However, the mechanism of TRIP13 influencing the invasion and metastasis of NSLCL has not been fully elucidated. Epithelial-mesenchymal transformation (EMT) serves an important role in tumor metastasis by enhancing the motility of cancer cells (21). At present, the mechanism of TRIP13 influencing EMT process remains unknown and should be further investigated.

The present study aimed to examine the association of TRIP13 with NSCLC. Reverse transcription-quantitative (RT-qPCR) and western blot analyses were conducted to assess the role of TRIP13 in regulating apoptosis, proliferation, invasion and migration. Furthermore, knockdown or overexpression of TRIP13 may affect the EMT during the progression of tumors. Studying the molecular biological mechanism underlying the occurrence and development of NSCLC and identifying effective biomarkers are extremely important.

\section{Materials and methods}

Clinical information and bioinformatic analyses. The clinical information of patients and mRNA expression levels of TRIP13 were obtained for NSCLC tissues $(n=515)$ and adjacent normal tissues $(n=59)$ from The Cancer Genome Atlas (TCGA) database (https://cancer.gov/). These tissues were obtained from 238 men and 276 women. The age of patients ranged from 40-80 years. The relationships between TRIP13 and different stages of NSCLC, as well as numbers of axillary lymph node metastases are presented. Kaplan-Meier analysis was performed to evaluate the prognostic value of TRIP13 at high level $(n=124)$ compared with low level $(n=378)$ according to data from TCGA database. The threshold of optimal gene expression was set as the cut-off. The log-rank test was used to further evaluate the P-value.

Cell culture and cell transfection. Human NSCLC cell lines, A549, H1299 and H661, were purchased from the Chinese Academy of Sciences. Cells were cultured in DMEM (Gibco; Thermo Fisher Scientific, Inc.) supplemented with 10\% FBS (Cell Sciences) in a humidified atmosphere with $5 \% \mathrm{CO}_{2}$ at $37^{\circ} \mathrm{C}$. Small interfering (si)RNA negative control (si-NC) and TRIP13 siRNA molecules (si1 and si2), pcDNA-3.1 vector (empty vector) and TRIP13-overexpressing plasmid were purchased from Suzhou GenePharma Co., Ltd. The cells were maintained in 6-well plates until they reached $60-70 \%$ confluence. According to the manufacturer's instructions, the siRNAs $(2.5 \mu \mathrm{g})$ and overexpression plasmid $(2.5 \mu \mathrm{g})$ were transfected into A549 and H1299 cells using Lipofectamine ${ }^{\circledR} 2000$ (Invitrogen; Thermo Fisher Scientific, Inc.) at room temperature and the medium with serum was changed after $6 \mathrm{~h}$. RNA was extracted $48 \mathrm{~h}$ after transfection to verify the knockdown and overexpression effects. All experiments were repeated three times. The siRNA sequences were: si1, 5'-GACCAG
AAAUGUGCAGUCU-3'; si2, 5'-GCAAAUCACUGGGUU CUAC-3'; and si-NC, 5'-UUCUCCGAACGUGUCACGUTT-3'.

$R T-q P C R$. Total RNA was extracted from NSCLC cell lines and tumor tissues using TRIzol ${ }^{\circledR}$ reagent (Invitrogen; Thermo Fisher Scientific, Inc.). Then, the RNA was reverse transcribed into cDNA using PrimeScript RT Master mix (Takara Bio, Inc.). The RT conditions were $37^{\circ} \mathrm{C}$ for $15 \mathrm{~min}$, $85^{\circ} \mathrm{C}$ for $5 \mathrm{sec}$ and $4^{\circ} \mathrm{C}$ preservation. The expression level of TRIP13 was determined via RT-qPCR using SYBR Green Master mix (Vazyme Biotech Co., Ltd.) and a Bio-Rad Real-Time PCR detection system (Bio-Rad Laboratories, Inc.). The complete thermocycling conditions were: Initial denaturation at $95 \mathrm{C}^{\circ}$ for $2 \mathrm{~min}$, followed by 40 cycles of denaturation at $95^{\circ} \mathrm{C}$ for $10 \mathrm{sec}$, annealing and elongation at $60^{\circ} \mathrm{C}$ for $30 \mathrm{sec}$, and the final extension at $72^{\circ} \mathrm{C}$ for $7 \mathrm{~min}$. $\beta$-actin was the internal control. The expression level of the relevant genes was calculated using the formula $2^{-\Delta \Delta \mathrm{Cq}}(22)$. All experiments were repeated three times. The primer sequences were as follows: TRIP13 forward, 5'-GCGTGGTCAATGCTGTCTTG-3' and reverse, 5'-CACGTCGATCTTCTCGGTGA-3' and $\beta$-actin forward, 5'-CCAACCGCGAGAAGATGA-3' and reverse, 5'-CCAGAGGCGTACAGGGATAG-3'.

Immunohistochemistry (IHC). Paraffin-embedded NSCLC tissues $(n=10)$ were fixed with $4 \%$ paraformaldehyde for $24 \mathrm{~h}$ at room temperature, then cut into 5- $\mu \mathrm{m}$ paraffin sections and attached to slides. After dewaxing with xylene for $30 \mathrm{~min}$ at room temperature, the slices were boiled for $10 \mathrm{~min}$ in citric acid buffer solution $(\mathrm{pH}$ 6.0) for antigen repair, blocked with $10 \%$ non-immune goat serum (LMAI Bio) for $30 \mathrm{~min}$ at room temperature and incubated with an antibody against TRIP13 (1:1,000; cat. no. ab204331; Abcam) overnight at room temperature. Then, the slices were incubated with HRP-conjugated secondary antibody $(1: 2,000$; cat. no. ab205718; Abcam) for $30 \mathrm{~min}$ at room temperature. After diaminobenzidine staining, the degree of staining was observed under a fluorescent microscope. There was a total of 10 patients, consisting of 5 men and 5 women. The age of patients ranged 44-70 years. The following samples were collected: Five tissues in right upper lobe, five tissues in right lower lobe, one tissue in left upper lobe and one tissue in left lower lobe. Moreover, the paired adjacent normal tissues were also obtained from these patients. All tissues were obtained between March 2020 and November 2020. All samples came from patients at The Third People's Hospital of Nantong Affiliated to Nantong University. Informed consent was obtained from each patient. This study was approved by the Ethics Committee of The Third People's Hospital of Nantong Affiliated to Nantong University. All experiments were repeated three times.

Cell apoptosis assay. The apoptotic rate was measured via flow cytometry and included the percent of early and late apoptotic cells. After transfection, the dead cells were collected and stained with $5 \mu \mathrm{l}$ Annexin $\mathrm{V}$ PE and $5 \mu \mathrm{l}$ 7-AAD (BD Biosciences) in the dark at room temperature for $15 \mathrm{~min}$. In total, $400 \mu \mathrm{l}$ diluted binding buffer was added to each sample. Then, the apoptotic rate was measured using a FACSCalibur flow cytometer (BD Biosciences), and the results 
were analyzed using FlowJo software 10.7 (FlowJo LLC). All experiments were repeated three times.

Cell cycle assay. Human A549 and H1299 cells were transfected with the siRNAs or overexpression plasmid for $48 \mathrm{~h}$. The cells were resuspended in $70 \%$ ethanol and incubated in a $-20^{\circ} \mathrm{C}$ freezer for $>24 \mathrm{~h}$. The samples were stained with $20 \mu \mathrm{g} / \mathrm{ml}$ PI and $20 \mu \mathrm{g} / \mathrm{ml}$ RNase A (Sigma-Aldrich; Merck KGaA) for $15 \mathrm{~min}$ at room temperature. Flow cytometry (BD Biosciences) was used to analyze cell cycle progression, and the data were processed using FlowJo software 10.7 (FlowJo LLC). All experiments were repeated three times.

Cell proliferation assay. The proliferation of the NSCLC cells was measured using a Cell Counting Kit-8 (CCK-8; Dojindo Molecular Technologies, Inc.) assay, according to the manufacturer's instructions. A total of $4 \times 10^{3}$ cells per well were seeded in 96-well plates and cultured for 0, 24, 48 and $72 \mathrm{~h}$. After incubation with $10 \mu \mathrm{lCCK}-8$ solution for $2 \mathrm{~h}$ at $37^{\circ} \mathrm{C}$, the absorbance values were measured at $450 \mathrm{~nm}$. All experiments were repeated three times.

Transwell assay. Transwell assays were performed to detect cell invasion and migration. For the invasion assay, a total of $40 \mu \mathrm{l}$ Matrigel (BD Biosciences) was diluted with serum-free medium and placed in the upper chamber (pore size, $3 \mu \mathrm{m}$; MilliporeSigma) for $30 \mathrm{~min}$ at $37^{\circ} \mathrm{C}$, and $2 \times 10^{5}$ cells were plated in the upper chamber with $200 \mu \mathrm{l}$ serum-free medium. DMEM with 20\% FBS was added to the lower chamber. For the migration assay, Matrigel was not required in the upper chamber. After incubation for $48 \mathrm{~h}$ at $37^{\circ} \mathrm{C}$, the cells that adhered to the lower surface of the filter were stained with $1 \%$ crystal violet for $10 \mathrm{~min}$ at room temperature. Then, the quantity of invading or migrating cells was determined under an Olympus IX73-FL-PH inverted microscope (Olympus Corporation). All experiments were repeated three times.

Wound healing assay. A549 and H1299 cells were seeded in a 6-well plate using medium with FBS-free for $24 \mathrm{~h}$ after transfection and covered $70-80 \%$ of the plate. A straight line was scratched the plate with a $10-\mu 1$ pipette tip and every line was spaced at $0.5 \mathrm{~cm}$. PBS was used to removed cell debris. The cell healing was filmed at 0 and $24 \mathrm{~h}$ using an Olympus IX73-FL-PH inverted microscope (Olympus Corporation). The distance of lines was measured using ImageJ 1.8.0 (National Institutes of Health). All experiments were repeated three times.

Western blotting. Total proteins were extracted from cells with RIPA lysis buffer (Beyotime Institute of Biotechnology), and the concentrations of the proteins were measured using a BCA protein assay kit (Beyotime Institute of Biotechnology). The samples were mixed with loading buffer, were electrophoresed on 10\% SDS-PAGE gels and transferred to PVDF membranes (MilliporeSigma). The membranes were blocked with 5\% non-fat milk (Beyotime Institute of Biotechnology) for $2 \mathrm{~h}$ at room temperature and incubated overnight at $4{ }^{\circ} \mathrm{C}$ with primary antibodies [anti-TRIP13, anti- $\beta$-actin, anti-vimentin, anti-E-cadherin, anti-snail family transcriptional repressor 1 (Snail)]. The membranes were completely washed three times with TBS-Tween-20 (0.1\%) (Beyotime Institute of Biotechnology) and further incubated with a specific HRP-conjugated secondary antibody $(1: 2,000$; cat. no. ab205718; Abcam) for $1 \mathrm{~h}$ at room temperature. The densitometry was measured using ImageJ 1.8.0. All experiments were repeated three times. The primary antibodies were anti-TRIP13 (1:1,000; cat. no. ab204331; Abcam), anti- $\beta$-actin (1:2,000; cat. no. ab8227; Abcam), anti-vimentin (1:1,000; cat. no. 5741S; Cell Signaling Technology,Inc.), anti-E-cadherin (1:1,000; cat. no. 3195S; Cell Signaling Technology, Inc.) and anti-Snail (1:1,000; cat. no. NBP2-27293; Novus Biologicals, Inc.).

Statistical analysis. All the data are presented as the mean \pm SD. Most of the differences between two groups were determined using an unpaired t-test. The expression level of TRIP13 between NSCLC tissues and adjacent normal tissues detected via RT-qPCR was compared using a paired Student's t-test. Multiple comparison between the group was performed using one-way ANOVA and Tukey's test. $\mathrm{P}<0.05$ was considered to indicate a statistically significant difference. All the data statistical analyses were conducted using GraphPad Prism 8.0 (GraphPad Software Inc.).

\section{Results}

TRIPI3 is upregulated in NSCLC. To study the expression of TRIP13 in NSCLC, clinical data from TCGA, including data from 59 normal samples and 515 primary tumor samples, were analyzed. It was found that TRIP13 was upregulated in the NSCLC tissues compared with the adjacent normal tissues (Fig. 1A). The data demonstrated that the expression level of TRIP13 was more significantly increased with higher stages of NSCLC, and each stage was statistically significant compared with the normal group (Fig. 1B).

To further evaluate the effect of TRIP13 expression on prognosis, four cancer stages were identified based on the number of axillary lymph nodes affected. As the number of affected nodes increased, the expression level of TRIP13 was significantly upregulated in the NSCLC tissues compared with the normal tissues, and increased TRIP13 expression predicted poor prognosis (Fig. 1C). Furthermore, the survival analysis revealed that patients with high TRIP13 levels $(n=124)$ had poorer prognosis compared with those with low TRIP13 levels $(\mathrm{n}=378)$ (Fig. 1D).

IHC analysis identified that the nuclei were stained yellow brown or dark brown and that TRIP13 was located in the nucleus in 10 NSCLC tissues (Fig. 2A). The results of TRIP13 positive cells were assessed by two pathologists who did not know the tumor grade. In total, 10 high-power fields were observed and the positive percentage was calculated. The quantitative data are shown in Fig. 2B. To determine the biological function of TRIP13 in NSCLC cells, three cell lines, namely, A549, H1299 and H661 cell lines, were selected for the experiment (Fig. 2C). The expression level of TRIP13 was low in A549 cells, but its expression was high in H1299 cells.

Knockdown of TRIP13 inhibits the proliferation, invasion and migration and increases apoptosis of H1299 cells. si1 

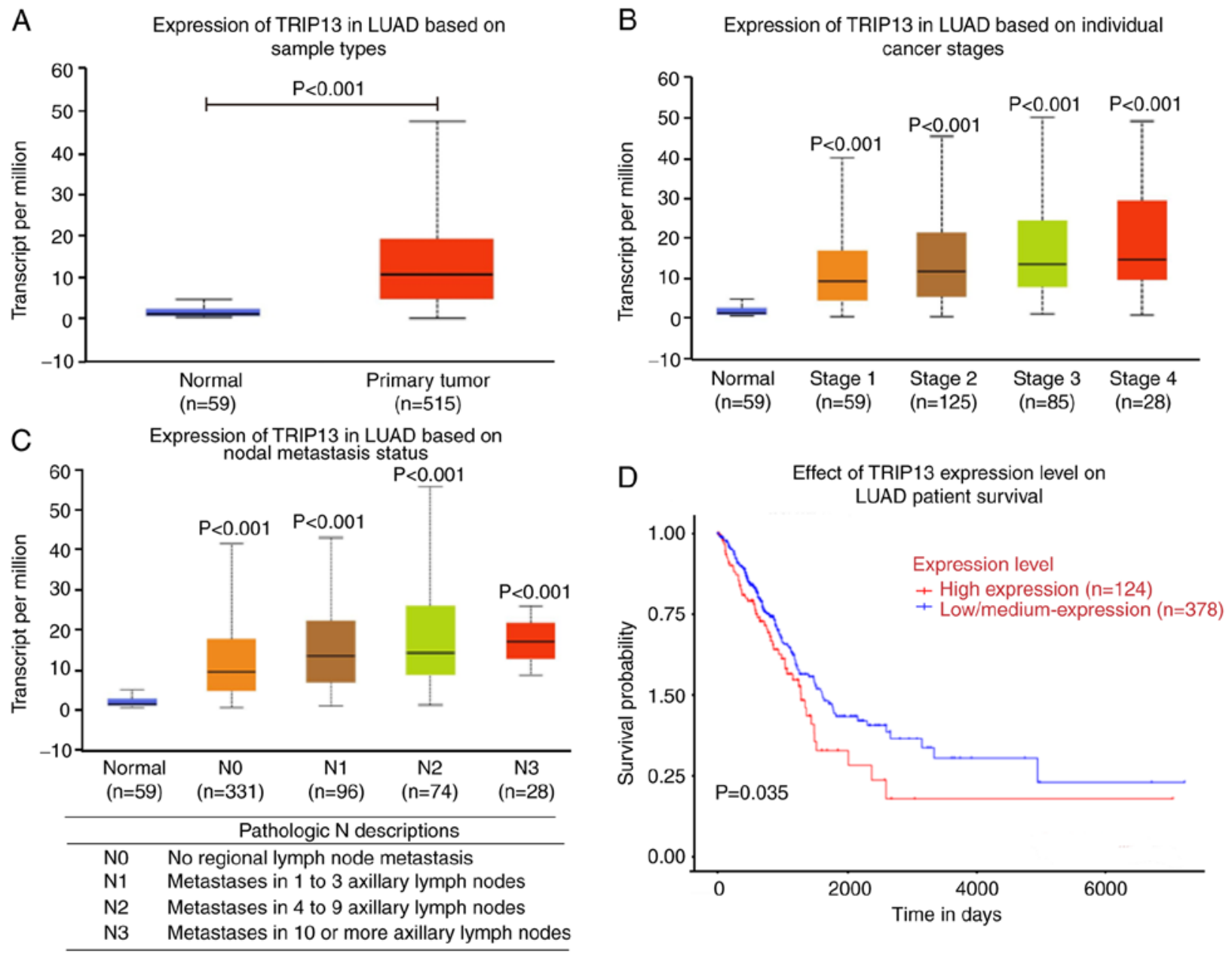

Figure 1. Expression level of TRIP13 in NSCLC. (A) TRIP13 was upregulated in NSCLC ( $\mathrm{n}=59$ ) compared with normal tissues ( $\mathrm{n}=515)$. (B) Expression level of TRIP13 in NSCLC based on individual cancer stages. (C) Relative TRIP13 expression in NSCLC based on the number of axillary lymph nodes. (D) The Kaplan-Meier Plotter analyzed the survival rate of patients with high or low levels of TRIP13. TRIP13, thyroid hormone receptor-interacting protein 13; NSCLC, non-small cell lung cancer; LUAD, lung adenocarcinoma.

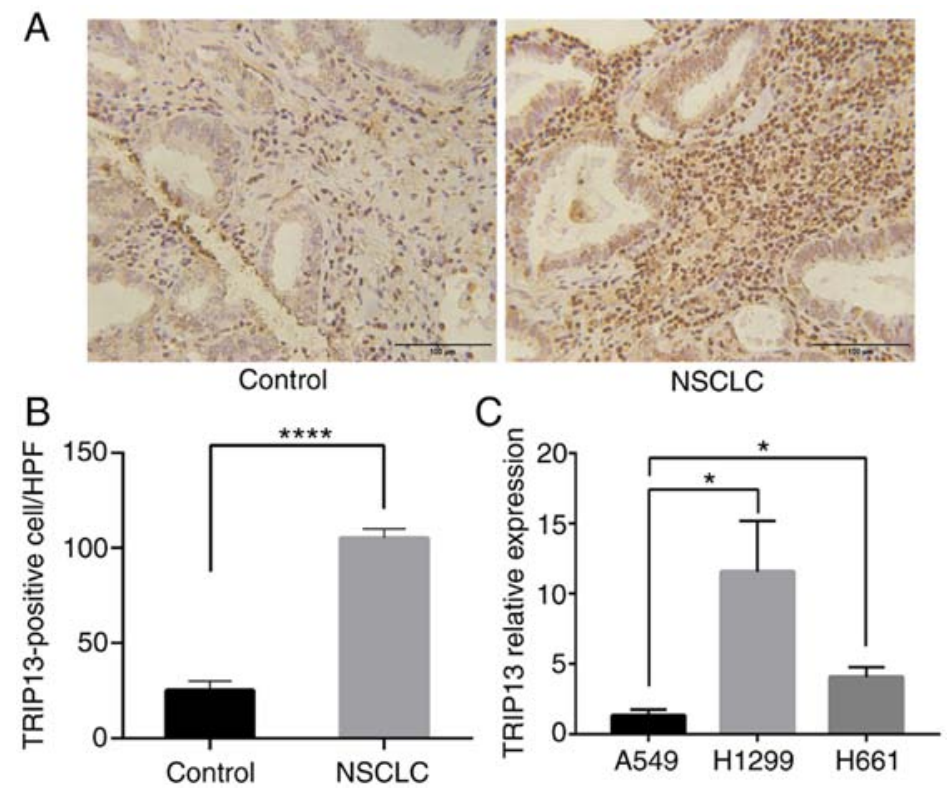

Figure 2. Expression level of TRIP13 in NSCLC tissues and cells. (A) Immunohistochemistry revealed the expression level of TRIP13 in NSCLC tissues compared with adjacent normal tissues $(\mathrm{n}=10)$. Scale bar, $100 \mu \mathrm{m}$. (B) Quantitative analysis of TRIP13 positive cells after staining was shown in 10 randomly selected HPF of view. (C) Reverse transcription-quantitative PCR was used to detect the expression level of TRIP13 in cell lines (A549, H1299, H661). All data are presented as the mean $\pm \mathrm{SD} .{ }^{*} \mathrm{P}<0.05,{ }^{* * * *} \mathrm{P}<0.001$. NSCLC, non-small cell lung cancer; TRIP13, thyroid hormone receptor-interacting protein 13 ; HPF, high-power-fields. 

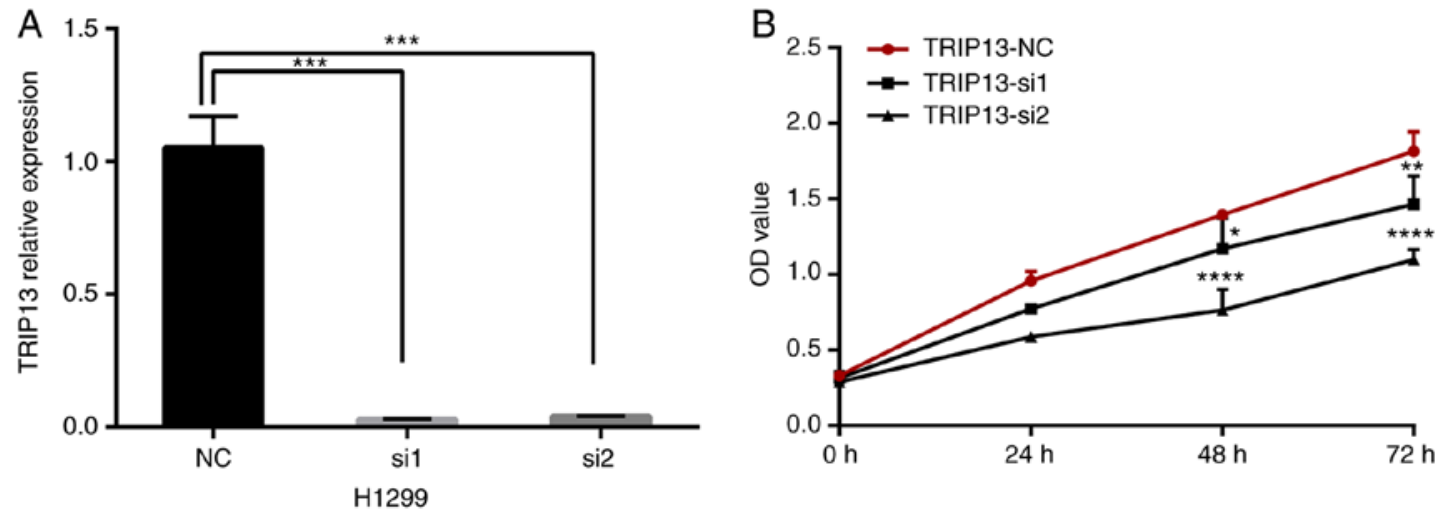

Figure 3. TRIP13 knockdown in H1299 cells. (A) Reverse transcription-quantitative PCR results showed the efficiency of TRIP13 mRNA expression knockdown. ${ }^{* * *} \mathrm{P}<0.001$. (B) Cell Counting Kit-8 assay measured the proliferation of $\mathrm{H} 1299$ cells at $450 \mathrm{~nm}$ at $0,24,48$ and $72 \mathrm{~h}$ after transfected with sil and si2 compared with NC group. ${ }^{*} \mathrm{P}<0.05,{ }^{* *} \mathrm{P}<0.01,{ }^{* * * * *} \mathrm{P}<0.001$ vs. NC group. All data are presented as the mean $\pm \mathrm{SD}$. TRIP13, thyroid hormone receptor-interacting protein 13; NC, negative control; siRNA, small interfering RNA; OD, optical density.

A

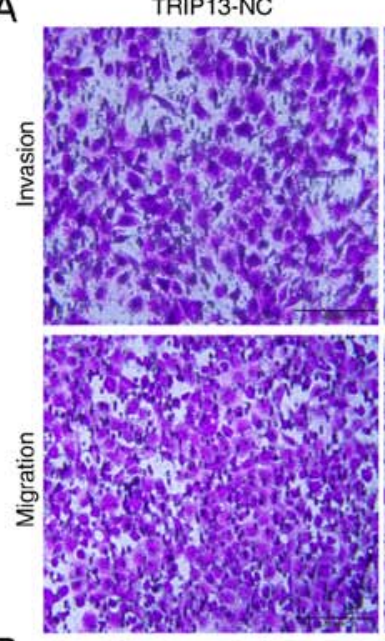

B
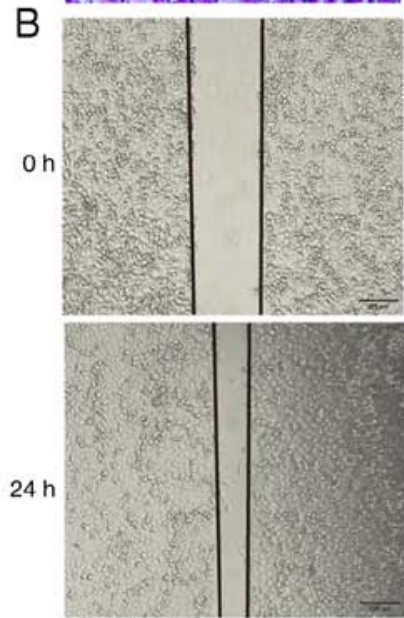

TRIP13-NC
TRIP13-si1
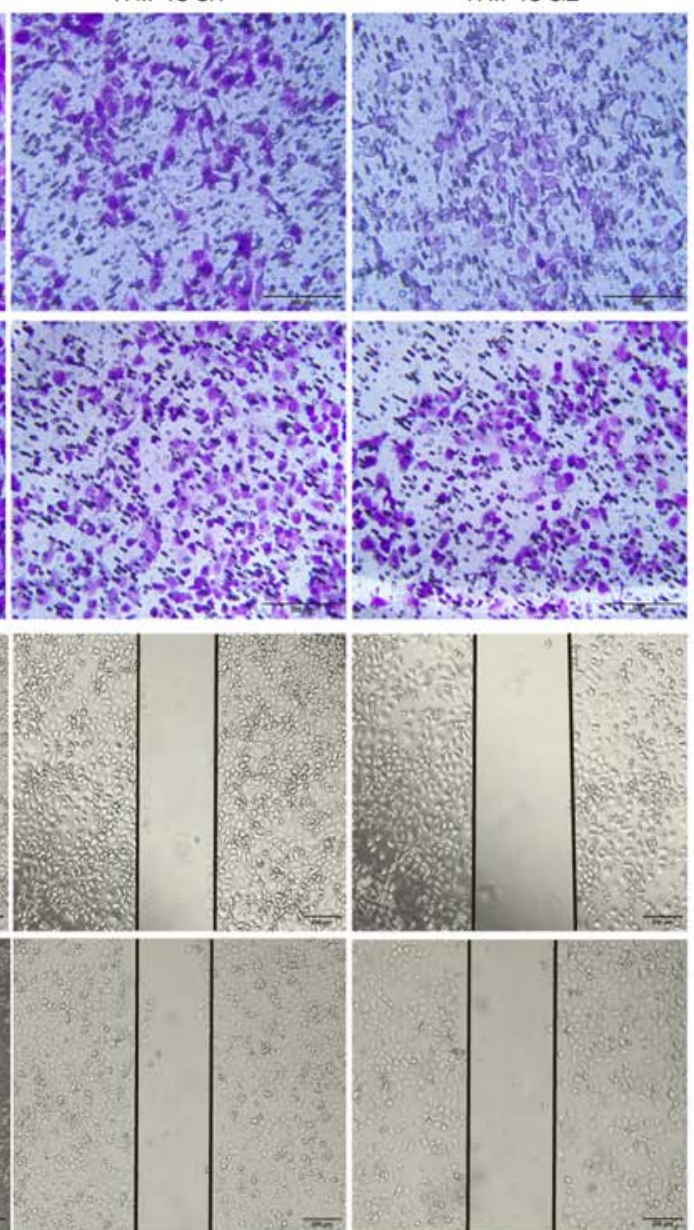

TRIP13-si1

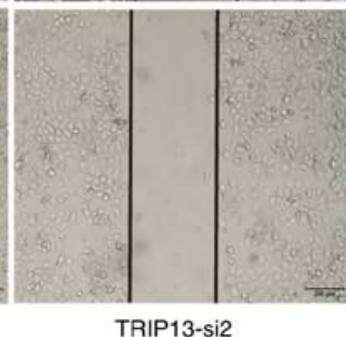

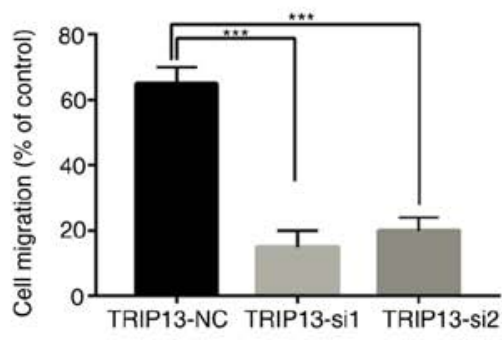

Figure 4. Knockdown of TRIP13 affects the migration and invasion of H1299 cells. (A) Transwell assay and the (B) scratch experiment was used to detect the migration and invasion in H1299 cells. Scale bar, $200 \mu \mathrm{m}$. All data are presented as the mean \pm SD. ${ }^{* * *}$ P $<0.001$. TRIP13, thyroid hormone receptor-interacting protein 13; NC, negative control; siRNA, small interfering RNA.

and si2 were used to knockdown the expression of TRIP13 in H1299 cells, which had a higher endogenous TRIP13 expression than the other cell lines. The RT-qPCR results revealed that the knockdown efficiency of si1 and si2 was substantial, and that the expression of TRIP13 was knocked down
(Fig. 3A). To further study the cellular biological functions, a CCK-8 assay was used to examine the proliferative abilities of the cells at $0,24,48$ and $72 \mathrm{~h}$. The knockdown of TRIP13 in H1299 cells significantly suppressed proliferation compared with the NC group (Fig. 3B) from 24 to 72 h. Moreover, the 

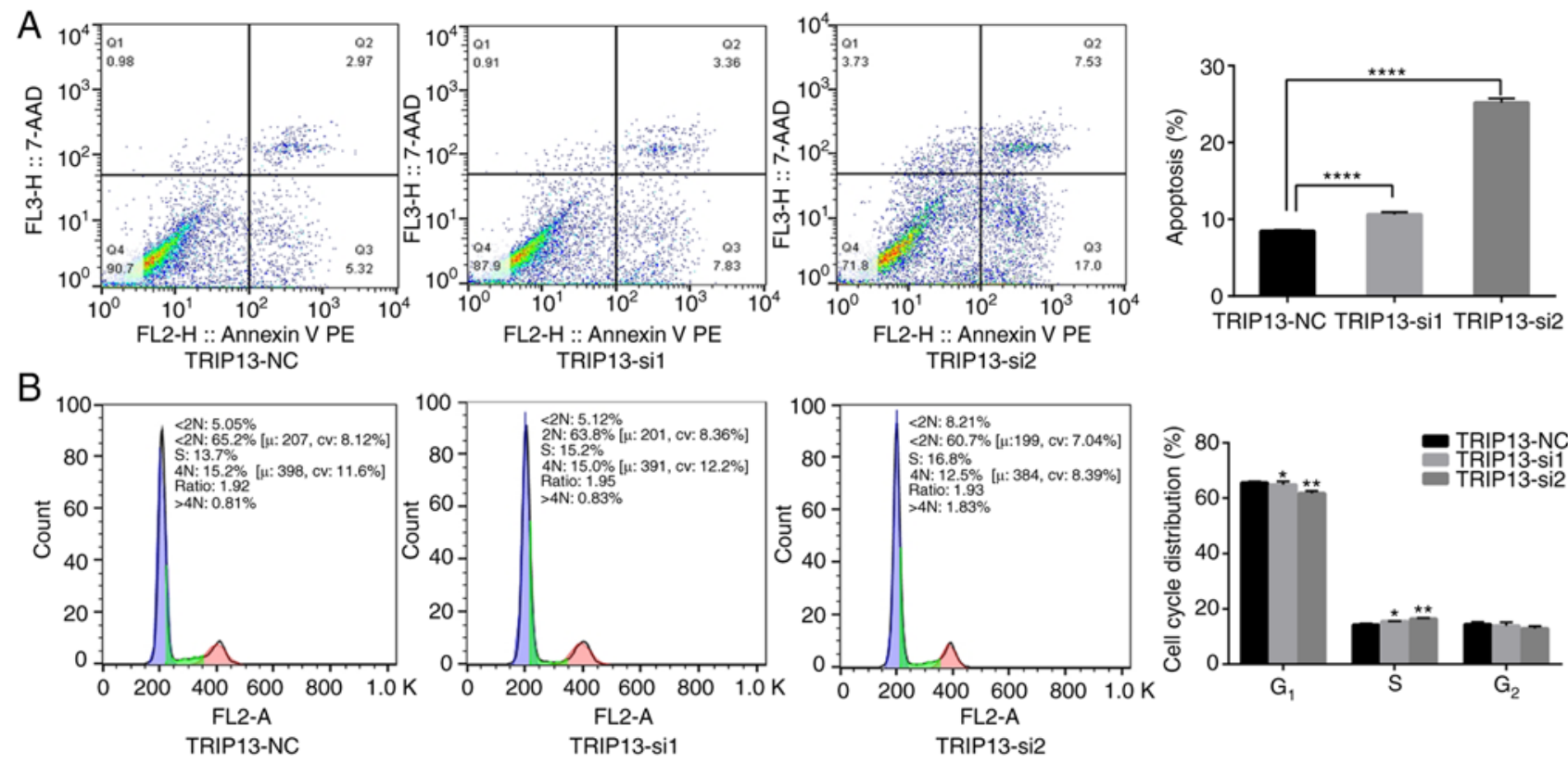

Figure 5. Knockdown of TRIP13 affects the apoptosis and cell cycle of H1299 cells after $48 \mathrm{~h}$. (A) Flow cytometry analyzed the cell apoptotic rate after transfected with si1 and si2 compared with NC group. ${ }^{* * * * *} \mathrm{P}<0.001$ vs. NC group. (B) Flow cytometry analyzed cell cycle distribution after transfected with si 1 and si 2 compared with $\mathrm{NC}$ group. ${ }^{*} \mathrm{P}<0.05,{ }^{* *} \mathrm{P}<0.01$ vs. $\mathrm{NC}$ group. All data are presented as the mean $\pm \mathrm{SD}$. TRIP13, thyroid hormone receptor-interacting protein 13; NC, negative control; siRNA, small interfering RNA.
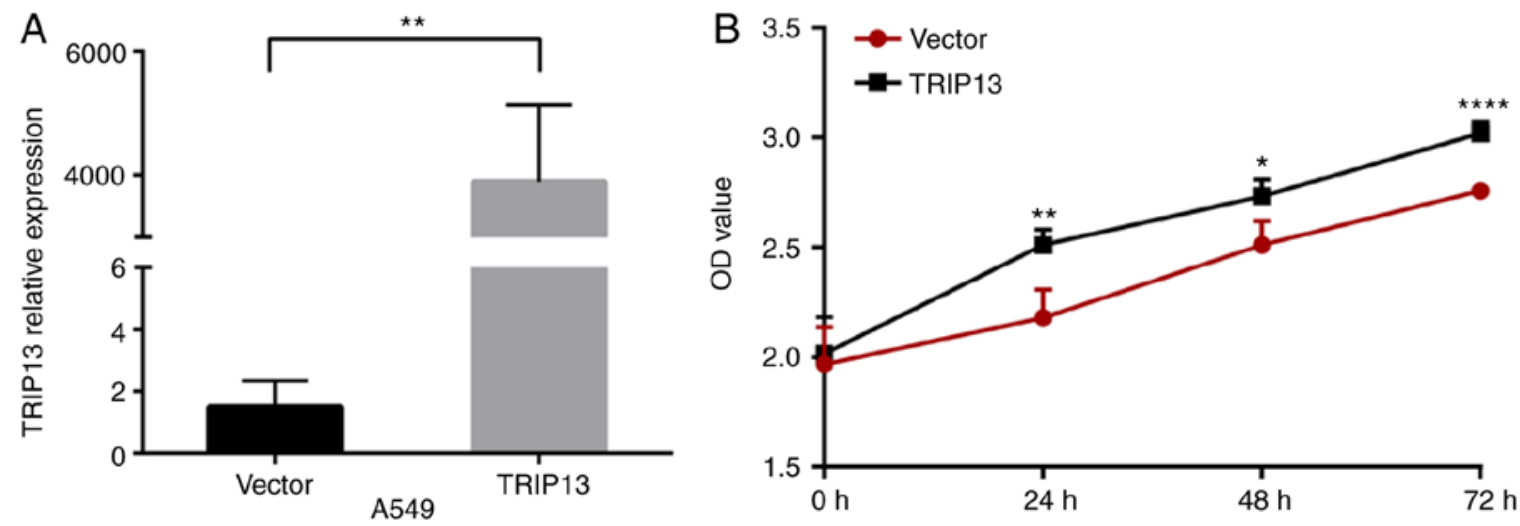

Figure 6. TRIP13 overexpression in A549 cells. (A) Reverse transcription-quantitative PCR results showed the efficiency of TRIP13 mRNA expression overexpression. ${ }^{* * *} \mathrm{P}<0.01$. (B) Cell Counting Kit-8 assay measured the viability of A549 cells at $450 \mathrm{~nm}$ at $0,24,48$ and $72 \mathrm{~h}$ after transfected with plasmid compared with vector group. ${ }^{*} \mathrm{P}<0.05,{ }^{* *} \mathrm{P}<0.01,{ }^{* * * *} \mathrm{P}<0.001$ vs. vector group. All data are presented as the mean $\pm \mathrm{SD}$. TRIP13, thyroid hormone receptor-interacting protein 13; OD, optical density.

knockdown of TRIP13 inhibited the H1299 cell migratory and invasive abilities, as evaluated using the Transwell assay (Fig. 4A). The wound healing experiment effectively detected lateral cell migration. The migratory ability of the knockdown group was significantly decreased compared with the NC group, as determined by comparing the gaps in the wound healing assay (Fig. 4B).

Flow cytometry accurately detected cellular apoptosis and the cell cycle progression. The change in the apoptotic rate could be one of the mechanisms that affect the malignant progression of NSCLC. The results indicated that knockdown of TRIP13 increased the rate of apoptosis in H1299 cells compared with the NC at $48 \mathrm{~h}$ after transfection (Fig. 5A). In addition, the distribution of the cell cycle reflected the stage of DNA synthesis in cancer cells. In the
TRIP13 knockdown group, there was a significant decrease in the number of cells in the $\mathrm{G}_{1}$ phase; on the other hand, TRIP13 knockdown induced H1299 cell cycle arrest in the $S$ phase and caused no changes in the number of cells in the $\mathrm{G}_{2}$ phase (Fig. 5B).

TRIP13 overexpression inhibits apoptosis and promotes proliferation, invasion and migration of $A 549$ cells. The aforementioned experimental results demonstrated that knockdown of TRIP13 significantly affected the function of NSCLC cells. In order to prove the role of TRIP13 in promoting cancer progression more effectively, the changes in abilities of cell apoptosis, proliferation, invasion and migration after overexpression of TRIP3 were detected. The transfection efficiency was determined via RT-qPCR, and 

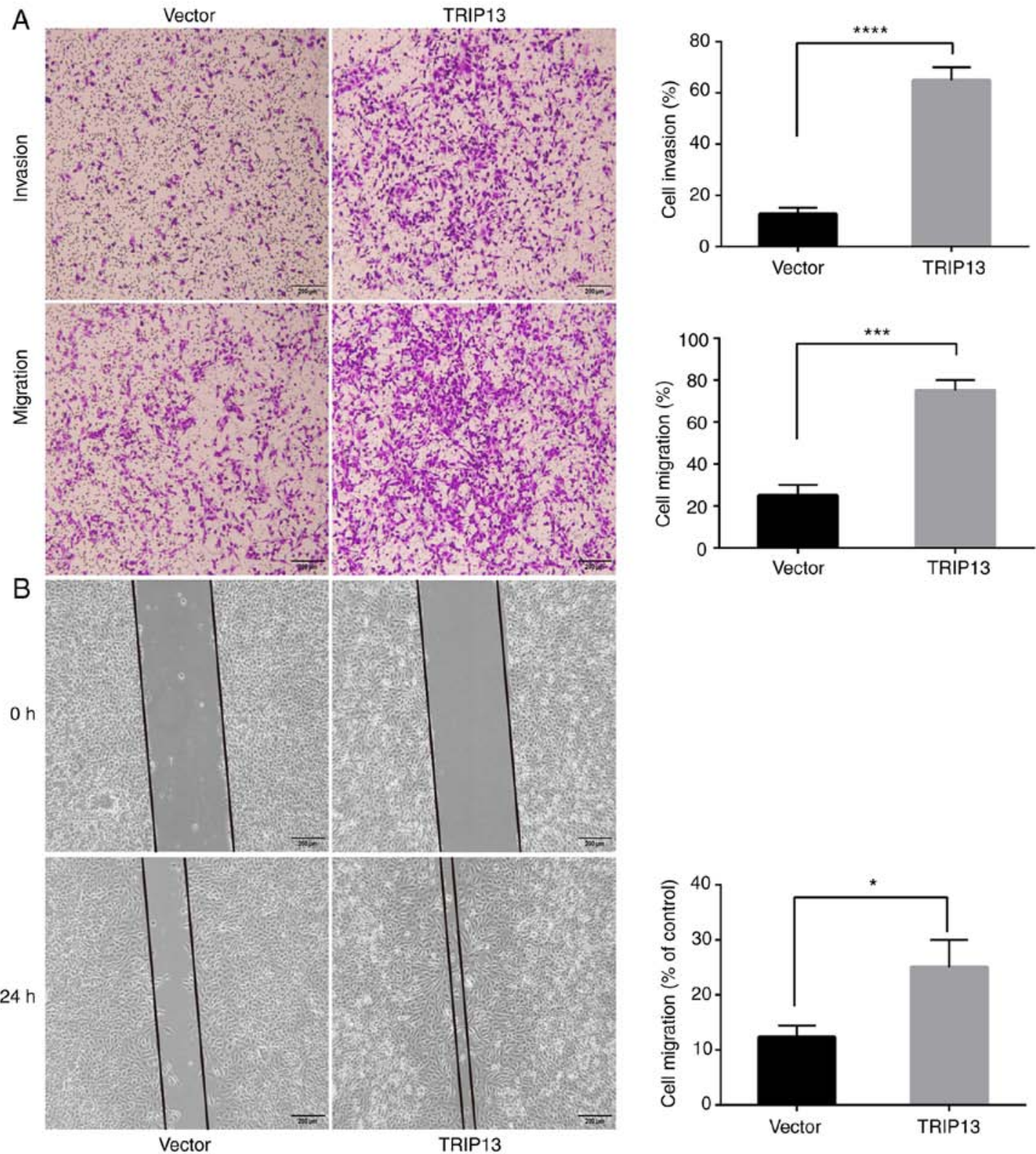

Figure 7. Overexpression of TRIP13 affects the migration and invasion of A549 cells. (A) Transwell assay and the (B) scratch experiment was used to detect the migration and invasion of A549 cells. Scale bar, $200 \mu \mathrm{m}$. All data are presented as the mean \pm SD. ${ }^{*} \mathrm{P}<0.05,{ }^{* * * *} \mathrm{P}<0.001,{ }^{* * * * *} \mathrm{P}<0.001$. TRIP13, thyroid hormone receptor-interacting protein 13 .

it was found that TRIP13 was significantly overexpressed (Fig. 6A). The TRIP13 overexpression group had increased cell proliferation compared with the vector group (Fig. 6B). The Transwell assay was used for detecting the H1299 cell migratory and invasive abilities. The results demonstrated that these abilities were significantly increased in the TRIP13 overexpression group compared with the vector group (Fig. 7A). Moreover, the wound healing experiment identified that TRIP13 overexpression significantly promoted lateral cell migration (Fig. 7B).

In contrast to TRIP13 knockdown, TRIP13 overexpression in A549 cells consistently reduced the apoptotic rate at $48 \mathrm{~h}$ after transfection (Fig. 8A). In addition, the TRIP13 overexpression group showed a significant decrease in the number of cells in the $S$ phase. However, the number of cells in the $G_{1}$ and $\mathrm{G}_{2}$ phase were markedly increased (Fig. 8B).
TRIP13 induces migration and invasion via EMT in NSCLC cells. A large number of studies have reported that EMT was an important biological process which epithelial-derived malignant tumor cells acquire the ability to migrate and invade (23-25). Western blotting was used to analyze the expression levels of several EMT-related factors, such as the epithelial marker E-cadherin and the mesenchymal markers vimentin and Snail, after transfection (Fig. 9A). The results demonstrated that TRIP13 knockdown could increase E-cadherin expression and decrease vimentin and Snail expression in H1299 cells. However, TRIP13 overexpression led to the opposite results. TRIP13 overexpression decreased the protein expression level of E-cadherin and enhanced the protein expression levels of vimentin and Snail (Fig. 9). The gray scale ratio of the three proteins to the internal reference was used to reflect the differences between each group (Fig. 9B and C). 

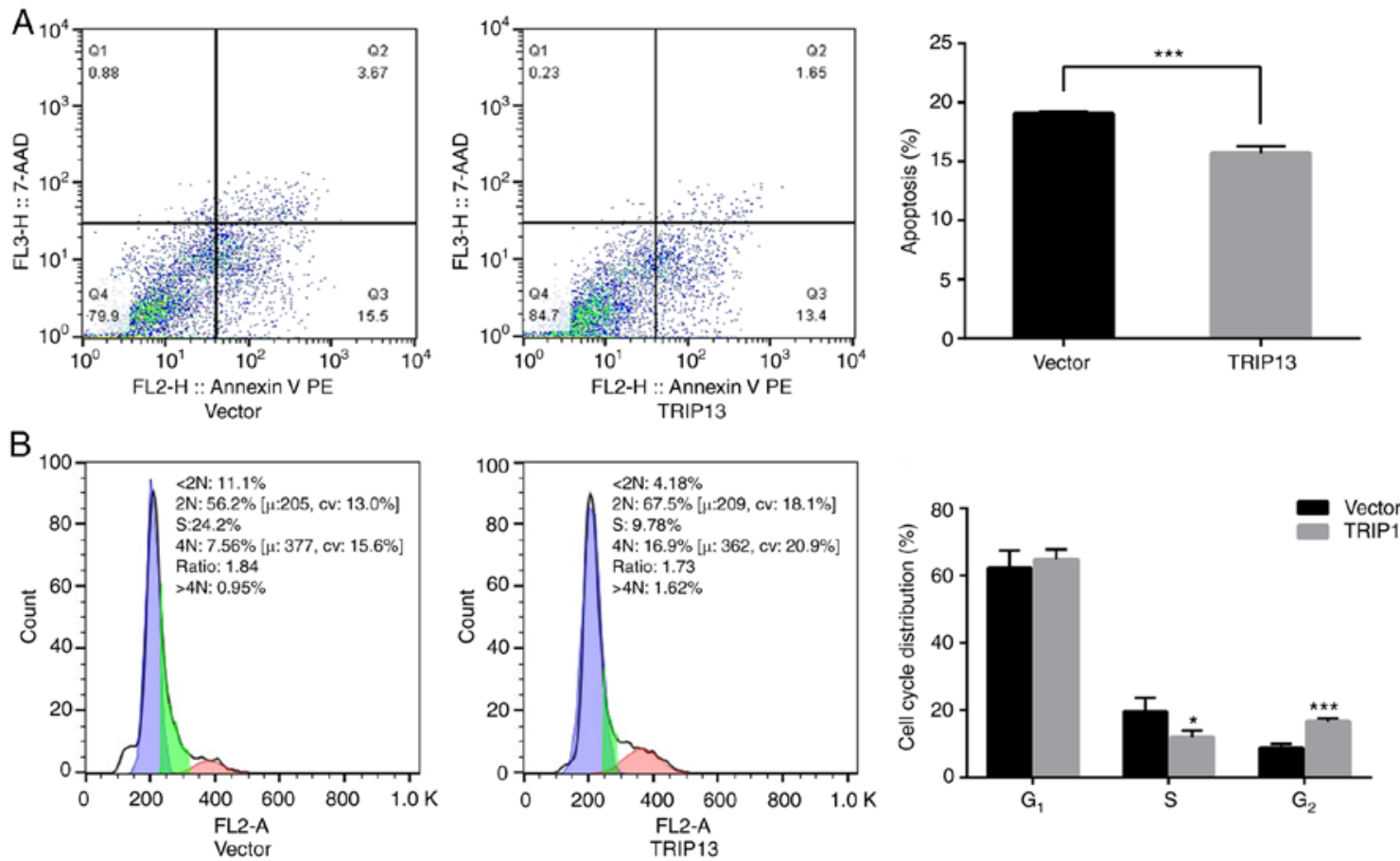

Figure 8. Overexpression of TRIP13 affects the apoptosis and cell cycle of A549 cells after $48 \mathrm{~h}$. (A) Flow cytometry analyzed the cell apoptosis rate after transfected with plasmid compared with vector group. ${ }^{* * *} \mathrm{P}<0.001$ vs. vector group. (B) Flow cytometry analyzed cell cycle distribution after transfected with plasmid compared with vector group. ${ }^{*} \mathrm{P}<0.05,{ }^{* * *} \mathrm{P}<0.001$ vs. vector group. All data are presented as the mean $\pm \mathrm{SD}$. TRIP13, thyroid hormone receptor-interacting protein 13 .

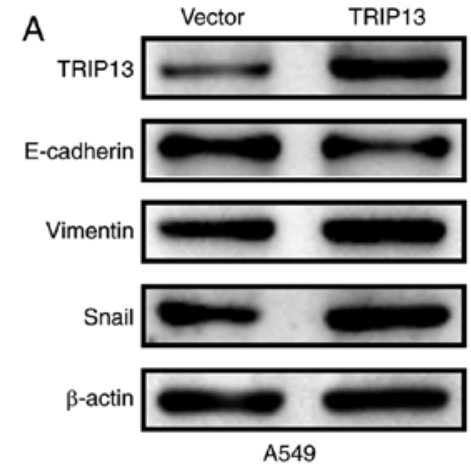

B
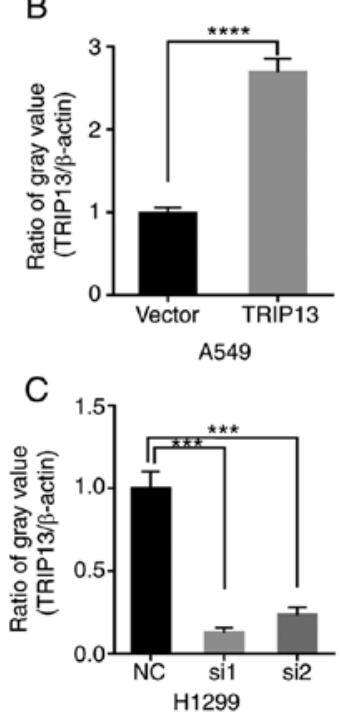
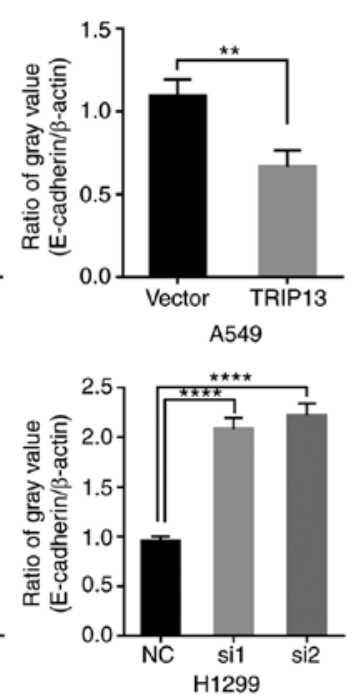

NC

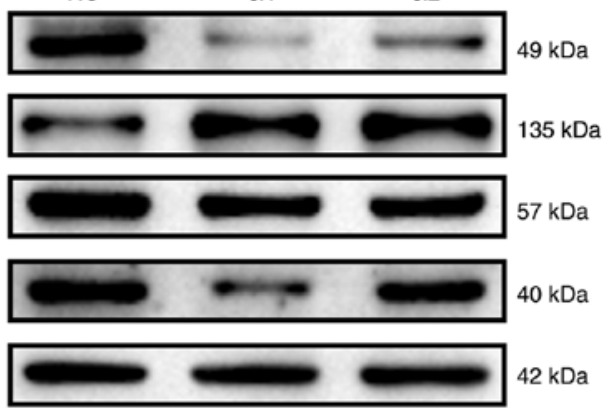

H1299
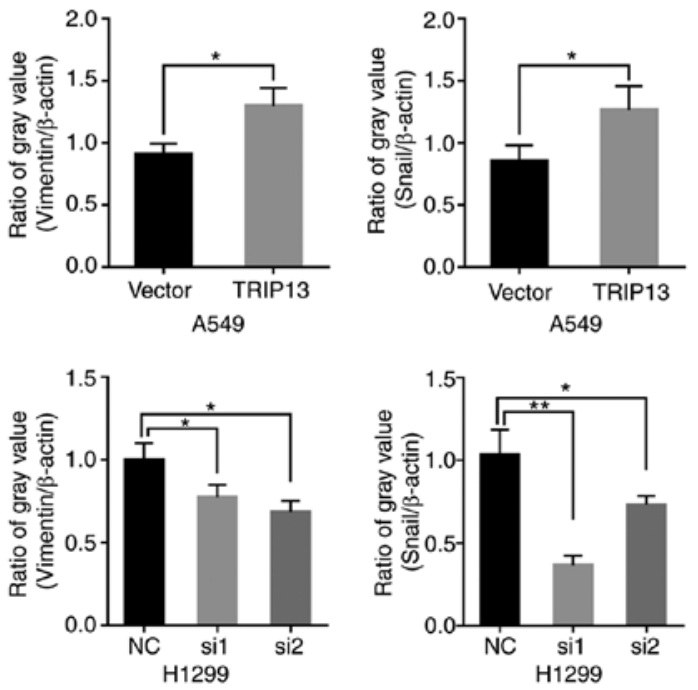

Figure 9. Knockdown and overexpression of TRIP13 affects EMT progression. (A) Western blotting results demonstrated that the knockdown or overexpression of TRIP13 influenced the expression levels of the EMT-related factors E-cadherin, Vimentin and Snail. Analysis of the expression levels of related proteins in (B) A549 and (C) H1299 cells. All data are presented as the mean \pm SD. ${ }^{*} \mathrm{P}<0.05,{ }^{* *} \mathrm{P}<0.01,{ }^{* * * *} \mathrm{P}<0.001,{ }^{* * * *} \mathrm{P}<0.001$. TRIP13, thyroid hormone receptor-interacting protein 13; NC, negative control; siRNA, small interfering RNA; Snail, snail family transcriptional repressor 1; EMT, epithelial-mesenchymal transformation. 


\section{Discussion}

China has a high incidence of lung cancer, and due to its poor prognosis, effective diagnostic criteria and treatment options are important for patients. The present study considered TRIP13 to be a potential biomarker worthy of further investigation.

Accumulating evidence has suggested that TRIP13 serves a carcinogenic role in numerous cancer types. For example, Zhang et al (26) reported that TRIP13 silencing could suppress the function of glioblastoma cells by regulating the expression of F-box and WD repeat domain containing 7 (FBXW7). c-Myc is a proto-oncogene that can affect the development of several tumor cells, and it has been shown that the TRIP13/FBXW7/ c-Myc pathway may provide a reliable target for glioblastoma treatment. In hepatocellular carcinoma, TRIP13 was positively correlated with actinin $\alpha 4$ and activated the Akt/mTOR pathway to accelerate the malignant progression of hepatocellular carcinoma (27). The mitotic checkpoint complex (MCC) coordinates with the SAC to maintain genomic stability and inhibits the expression of certain complexes, such as anaphase promoting complex (28). TRIP13 bound to p31comet to transform activate closed mitotic arrest deficient 2 like 1 (MAD2) into open MAD2, which was not active and could cause the decomposition of MCC. TRIP13 was further shown to be a AAA+ ATPase that binds to related proteins (29-31).

As the use of chemotherapeutic drugs has increased in the treatment of tumors, cancer cells have become more resistant. A previous study reported that upregulation of TRIP13 could enhance the progression of bladder cancer cells. These authors revealed that the proliferation of tumor cells was significantly decreased after knockdown of TRIP13. Moreover, the apoptotic rate was also significantly reduced in these drug-resistant cells, as shown by flow cytometry (18). To further investigate the mechanism via which TRIP13 participates in DNA repair, the researchers detected the expression level of $\mathrm{H} 2 \mathrm{AX}$ phosphorylated at serine $139(\gamma \mathrm{H} 2 \mathrm{AX})$, which is a typical marker of DNA damage, and RAD50 double strand break repair protein (RAD50), which is a typical marker of DNA repair. The results showed that overexpression of TRIP13 could inhibit the expression of $\gamma \mathrm{H} 2 \mathrm{AX}$ and enhance the expression of RAD50 after treatment with cisplatin. Thus, these researchers suggested that TRIP13 may promote DNA repair in tumor cells (32). Therefore, TRIP13 could be considered a novel potential target for the diagnosis and treatment of tumors.

In the present study, a high expression of TRIP13 in NSCLC was identified. TRIP13 was differentially expressed in NSCLC of different grades, based on the database results, and indicated a poor prognosis. Furthermore, knockdown of TRIP13 in H1299 cells increased apoptosis and inhibited proliferation, invasion and migration. Consistently, overexpression of TRIP13 inhibited apoptosis and promoted proliferation, invasion and migration of A549 cells.

EMT is closely associated with the aggressive behavior of cancer cells (33). E-cadherin is responsible for cell adhesion and cell cytoskeleton organization. The loss of E-cadherin is a key step in the EMT process, leading to the transition of epithelial cells to aggressive mesenchymal cells. It has also been confirmed that E-cadherin promotes cell proliferation and polarization to accelerate the metastasis of tumors (34). Moreover, changes in vimentin expression are closely associated with the malignant progression of tumors (35). As an important component of the signaling pathway, Snail promotes the progression of NSCLC (36). Previous studies have reported that TRIP13 induced changes in the expression levels of several EMT markers to promote the progression of several cancer types $(32,37,38)$.

However, there are some limitations in the present study. It has been proved that TRIP13 could affect the metastasis of NSCLC via EMT pathways. However, the other pathways that TRIP13 could affect metastasis are yet to be further determined. The relationship between TRIP13 and prognosis of patients has not been evaluated. These were the major points for the future work.

In conclusion, the present study demonstrated that upregulated TRIP13 could be a novel and potential biomarker for the diagnosis and treatment of NSCLC and served an important role in the malignant progression of NSCLC.

\section{Acknowledgements}

Not applicable.

\section{Funding}

This study was supported by the Health Bureau of Nantong City (grant no. WKZL2018054) and the Nantong Science and Technology Bureau (grant no. MSZ18008).

\section{Availability of data and materials}

The datasets generated during the current study are available from The Cancer Genome Atlas dataset (https://cancer.gov/).

\section{Authors' contributions}

JS was devoted to the conception and design of this study. RL and QZ performed the experiments. LJ, LC and FW analyzed the experimental data and presented statistical results. RL, QZ and FW collected the tissues. RL, QZ and JS wrote the manuscript. JS contributed to revise this manuscript and approved the final version to be published. RL, QZ and JS were responsible for confirming the authenticity of the raw data. All authors read and agreed to be accountable for all aspects of the work in ensuring that questions related to the accuracy or integrity of any part of the work are appropriately investigated and resolved.

\section{Ethics approval and consent to participate}

Informed consent was obtained from each patient. This study was approved by the Ethics Committee of The Third People's Hospital of Nantong Affiliated to Nantong University.

\section{Patient consent for publication}

Not applicable.

\section{Competing interests}

The authors declare that they have no competing interests. 


\section{References}

1. Sung H, Ferlay J, Siegel RL, Laversanne M, Soerjomataram I Jemal A and Bray F: Global cancer statistics 2020: GLOBOCAN estimates of incidence and mortality worldwide for 36 cancers in 185 countries. CA Cancer J Clin 71: 209-249, 2012.

2. Bade B and Dela Cruz C: Lung cancer 2020: Epidemiology, etiology, and prevention. Clin Chest Med 41: 1-24, 2020.

3. Schuller HM: The impact of smoking and the influence of other factors on lung cancer. Expert Rev Respir Med 13: 761-769, 2019.

4. Rivera GA and Wakelee H: Lung cancer in never smokers. Adv Exp Med Biol 893: 43-57, 2016.

5. Jones GS and Baldwin DR: Recent advances in the management of lung cancer. Clin Med (Lond) 18 (Suppl 2): S41-S46, 2018.

6. Steven A, Fisher SA and Robinson BW: Immunotherapy for lung cancer. Respirology 21: 821-833, 2016.

7. Kim D, Lee YS, Kim DH and Bae SC: Lung cancer staging and associated genetic and epigenetic events. Mol Cells 43: 1-9, 2020

8. Skřičková J, Kadlec B, Venclíček O and Merta Z: Lung cancer. Cas Lek Cesk 157: 226-236, 2018.

9. Wang Y, Huang J, Li B, Xue H, Tricot G, Hu L, Xu Z, Sun X, Chang S, Gao L, et al: A small-molecule inhibitor targeting TRIP13 suppresses multiple myeloma progression. Cancer Res 80: 536-548, 2020.

10. Ye Q, Rosenberg S, Moeller A, Speir J, Su T and Corbett K TRIP13 is a protein-remodeling AAA+ ATPase that catalyzes MAD2 conformation switching. Elife 4: e07367, 2015.

11. Clairmont CS, Sarangi P, Ponnienselvan K, Galli LD, Csete I, Moreau L, Adelmant G, Chowdhury D, Marto JA and D'Andrea AD: TRIP13 regulates DNA repair pathway choice through REV7 conformational change. Nat Cell Biol 22: 87-96, 2020.

12. Yost S, de Wolf B, Hanks S, Zachariou A, Marcozzi C, Clarke M, de Voer R, Etemad B, Uijttewaal E, Ramsay E, et al: Biallelic TRIP13 mutations predispose to Wilms tumor and chromosome missegregation. Nat Genet 49: 1148-1151, 2017.

13. Ma HT and Poon RYC: TRIP13 regulates both the activation and inactivation of the spindle-assembly checkpoint. Cell Rep 14: 1086-1099, 2016.

14. Tao Y, Yang G, Yang H, Song D, Hu L, Xie B, Wang H, Gao L, Gao M, Xu H, et al: TRIP13 impairs mitotic checkpoint surveillance and is associated with poor prognosis in multiple myeloma. Oncotarget 8: 26718-26731, 2017.

15. Yao J, Zhang X, Li J, Zhao D, Gao B, Zhou H, Gao S and Zhang L: Silencing TRIP13 inhibits cell growth and metastasis of hepatocellular carcinoma by activating of TGF- $\beta 1 / \mathrm{smad} 3$. Cancer Cell Int 18: 208, 2018.

16. Ju L, Li X, Shao J, Lu R, Wang Y and Bian Z: Upregulation of thyroid hormone receptor interactor 13 is associated with human hepatocellular carcinoma. Oncol Rep 40: 3794-3802, 2018.

17. Sheng N, Yan L, Wu K, You W, Gong J, Hu L, Tan G, Chen H and Wang Z: TRIP13 promotes tumor growth and is associated with poor prognosis in colorectal cancer. Cell Death Dis 9: 402, 2018

18. Gao Y, Liu S, Guo Q, Zhang S, Zhao Y, Wang H, Li T, Gong Y, Wang Y, Zhang T, et al: Increased expression of TRIP13 drives the tumorigenesis of bladder cancer in association with the EGFR signaling pathway. Int J Biol Sci 15: 1488-1499, 2019.

19. Dong L, Ding H, Li Y, Xue D, Li Z, Liu Y, Zhang T, Zhou J and Wang P: TRIP13 is a predictor for poor prognosis and regulates cell proliferation, migration and invasion in prostate cancer. Int J Biol Macromol 121: 200-206, 2019.

20. Zhang Q, Dong Y, Hao S, Tong Y, Luo Q and Aerxiding P: The oncogenic role of TRIP13 in regulating proliferation, invasion, and cell cycle checkpoint in NSCLC cells. Int J Clin Exp Pathol 12: 3357-3366, 2019.
21. Mittal V: Epithelial mesenchymal transition in tumor metastasis. Annu Rev Pathol 13: 395-412, 2018.

22. Livak KJ and Schmittgen TD: Analysis of relative gene expression data using real-time quantitative PCR and the 2(-Delta Delta C(T)) method. Methods 25: 402-408, 2001

23. Pastushenko I and Blanpain C: EMT transition states during tumor progression and metastasis. Trends Cell Biol 29: 212-226, 2019.

24. Yeung KT and Yang J: Epithelial-mesenchymal transition in tumor metastasis. Mol Oncol 11: 28-39, 2017.

25. Saitoh M: Involvement of partial EMT in cancer progression. J Biochem 164: 257-264, 2018.

26. Zhang G, Zhu Q, Fu G, Hou J, Hu X, Cao J, Peng W, Wang X, Chen $\mathrm{F}$ and $\mathrm{Cui} \mathrm{H}$ : TRIP13 promotes the cell proliferation, migration and invasion of glioblastoma through the FBXW7/c-MYC axis. Br J Cancer 121: 1069-1078, 2019.

27. Zhu MX, Wei CY, Zhang PF, Gao DM, Chen J, Zhao Y, Dong SS and Liu BB: Elevated TRIP13 drives the AKT/mTOR pathway to induce the progression of hepatocellular carcinoma via interacting with ACTN4. J Exp Clin Cancer Res 38: 409, 2019.

28. Alfieri C, Chang L, Zhang Z, Yang J, Maslen S, Skehel M and Barford D: Molecular basis of APC/C regulation by the spindle assembly checkpoint. Nature 536: 431-436, 2016.

29. Alfieri C, Chang L and Barford D: Mechanism for remodelling of the cell cycle checkpoint protein MAD2 by the ATPase TRIP13. Nature 559: 274-278, 2018.

30. Ye Q, Kim D, Dereli I, Rosenberg SC, Hagemann G, Herzog F, Tóth A, Cleveland DW and Corbett KD: The AAA+ ATPase TRIP13 remodels HORMA domains through N-terminal engagement and unfolding. EMBO J 36: 2419-2434, 2017.

31. Lu S, Qian J, Guo M, Gu C and Yang Y: Insights into a crucial role of TRIP13 in human cancer. Comput Struct Biotechnol J 17: 854-861, 2019.

32. Lu S, Guo M, Fan Z, Chen Y, Shi X, Gu C and Yang Y: Elevated TRIP13 drives cell proliferation and drug resistance in bladder cancer. Am J Transl Res 11: 4397-4410, 2019.

33. Lu W and Kang Y: Epithelial-mesenchymal plasticity in cancer progression and metastasis. Dev Cell 49: 361-374, 2019.

34. Venhuizen J, Jacobs F, Span P and Zegers M: P120 and E-cadherin: Double-edged swords in tumor metastasis. Semin Cancer Biol 60: 107-120, 2020.

35. Strouhalova K, Přechová M, Gandalovičová A, Brábek J, Gregor $\mathrm{M}$ and Rosel D: Vimentin intermediate filaments as potential target for cancer treatment. Cancers (Basel) 12: 184, 2020.

36. Xie Q, Zhu Z, He Y, Zhang Z, Zhang Y, Wang Y, Luo J, Peng T, Cheng F, Gao J, et al: A lactate-induced Snail/STAT3 pathway drives GPR81 expression in lung cancer cells. Biochim Biophys Acta Mol Basis Dis 1866: 165576, 2020

37. Agarwal S, Behring M, Kim HG, Chandrashekar DS, Chakravarthi BVSK, Gupta N, Bajpai P, Elkholy A, Al Diffalha S, Datta PK, et al: TRIP13 promotes metastasis of colorectal cancer regardless of p53 and microsatellite instability status. Mol Oncol 14: 3007-3029, 2020.

38. Kurita K, Maeda M, Mansour MA, Kokuryo T, Uehara K, Yokoyama Y, Nagino M, Hamaguchi M and Senga T: TRIP13 is expressed in colorectal cancer and promotes cancer cell invasion. Oncol Lett 12: 5240-5246, 2016.

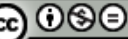

This work is licensed under a Creative Commons Attribution-NonCommercial-NoDerivatives 4.0 International (CC BY-NC-ND 4.0) License. 